\title{
Detection of Submillimetre Galaxies in the Lockman Hole using the European VLBI Network
}

\author{
Andy Biggs* \\ European Southern Observatory, Karl-Schwarzschild-Straße 2, D-85748 Garching, Germany \\ E-mail: abiggs@eso.org \\ Josh Younger \\ Harvard-Smithsonian Center for Astrophysics, 60 Garden Street, Cambridge, MA 02138, USA \\ E-mail: jyoungerecfa.harvard.edu
}

\section{Rob Ivison}

UK Astronomy Technology Centre, Royal Observatory, Blackford Hill, Edinburgh, EH9 3HJ, UK

E-mail: rjieroe.ac.uk

\begin{abstract}
Submillimetre Galaxies (SMGs) are high-redshift starbursts that are believed to be the precursors to modern-day giant elliptical galaxies. AGN appear to be an integral part of the evolution of these massive, star-forming galaxies; this is implied by the discovery of black holes at the centres of all nearby, massive galaxies and tracers of AGN activity have indeed been detected in a significant fraction of SMGs e.g. via deep X-ray observations and NIR spectroscopy, although they appear not to be bolometrically dominant. Radio observations offer an alternative, dust-unobscured, route to quantifying the contribution of AGN to the SMG luminosity, but only if the starburst contribution can be removed. The most effective way of doing this is with high-resolution VLBI which filters out the low-brightness extended emission typical of star forming regions leaving only the compact AGN cores. We have observed three SMGs in the Lockman Hole using the European VLBI Network at a wavelength of $18 \mathrm{~cm}$. All three are relatively bright ( $250 \mu \mathrm{Jy}$ ) and from MERLIN imaging were known to contain compact structure. Two of them are detected thus indicating that AGN are common in radio-detected SMGs and contribute significantly to the radio luminosity.
\end{abstract}

Panoramic Radio Astronomy: Wide-field 1-2 GHz research on galaxy evolution - PRA2009

June 02 - 052009

Groningen, the Netherlands

* Speaker. 


\section{Introduction}

Over the past twenty years it has become clear that supermassive black holes (SMBHs) in the nuclei of galaxies are common [6] and that their masses are strongly correlated with the properties of the stars in their host galaxies [4]. Recent theoretical models [3] have cast the co-evolution of SMBHs and galaxies in the context of a cosmic cycle driven by major mergers: high- $z$ gas-rich spiral galaxies collide, triggering a gas inflow that fuels a nuclear starburst and feedback-regulated SMBH growth which leads to a bright quasar, after which gas exhaustion and violent relaxation transform the remnant into a massive red elliptical galaxy.

These models of galaxy evolution predict that hyperluminous starbursts at high- $z$ will be associated with periods of active SMBH growth. Submillimetre galaxies (SMGs) provide an ideal laboratory for testing these models. These extreme objects are powered by mergers of gas-rich galaxies and are the likely progenitors of today's massive elliptical galaxies. Thus, SMGs are prime candidates for the transition objects that theory predicts should be powered by a mixture of star formation and active black hole growth.

To date, most observational searches for actively growing SMBHs in SMGs, including X-ray detections [1], infrared spectroscopy [7] and far-infrared morphology [8], have focused on largely indirect indicators. Very high-resolution radio imaging, by contrast, is capable of directly imaging any ultra-compact, luminous cores that can only be produced by active SMBHs.

\section{Sample and Observing Strategy}

The Lockman Hole is a region of the Northern sky with a remarkably low galactic HI column density that has been studied over the entire spectrum, including several submm surveys. Our sample of SMGs consists of those with VLA radio detections [5] within 6 arcmin of the pointing centre, $S_{1.4 \mathrm{GHz}}>50 \mu \mathrm{Jy}$ and for which MERLIN imaging indicates the presence of compact structure on scales of 250 mas [2]. Using the EVN, we have to date observed three SMGs in a bright sub-sample $\left(S_{1.4 \mathrm{GHz}}>250 \mu \mathrm{Jy}\right)$ with three fainter ones to be observed in June 2009.

Observations took place in June 2008 using 7 stations of the Western EVN including the 100$\mathrm{m}$ dish at Effelsberg, the 76-m Lovell telescope at Jodrell Bank and the 14 25-m dishes of the WSRT operating as a phased array. Data at a wavelength of $18 \mathrm{~cm}$ were recorded over a bandwidth of $64 \mathrm{MHz}$ per polarisation during two epochs each of $12 \mathrm{~h}$ duration (including calibration scans) resulting in final image sensitivities of approximately $10 \mu \mathrm{Jy} / \mathrm{beam}$. The naturally-weighted synthesised beam had a FWHM of 30 mas.

Due to the very small primary beam of the WSRT phased array, it was impossible to observe the entire 6-arcmin Effelsberg primary beam with a single pointing position. Instead we opted to point all telescopes apart from the WSRT at a single position whilst the WSRT cycled between the three SMGs. This maximised sensitivity on our primary targets whilst the use of short integration times and narrow channels gives useful sensitivity across the entire field (Fig. 1).

\section{Results}

Of the three SMGs that we primarily targeted, two were detected (Fig. 11). We have measured their flux densities and sizes and from these determine brightness temperatures in excess of several 


\begin{tabular}{cccc}
\hline Source & $S_{1.4 \mathrm{GHz}}(\mu \mathrm{Jy})$ & EVN/VLA $(\%)$ & $T_{b}\left(\times 10^{5} \mathrm{~K}\right)$ \\
\hline LOCKSMG1 & $315 \pm 11$ & 100 & 2.3 \\
LOCKSMG2 & $104 \pm 15$ & 40 & 7.5 \\
\hline
\end{tabular}

Table 1: Measured parameters of the two detected SMGs.

times $10^{5} \mathrm{~K}$ (Table 1) corrected for primary beam attenuation and redshift (measured spectroscopically in one case and assumed to have the average value for the SMGs in the other). These values are high enough that we can rule out anything other than an AGN as the source of the detected radio flux.

The extremely high resolution also allows us to separate the relative contribution made by star formation to the total radio luminosity of our targets, thus isolating the intrinsic luminosity of the AGN. We find that the AGN contributes $\approx 100 \%$ and $40 \%$ in each case. Combined with the known scaling of this quantity with SMBH mass this yields a lower limit for the mass of the SMBH in these systems. Our two targets have SMBHs with very high radio luminosities which suggest masses in the range 100 million to 1 billion suns. That these extremely massive SMBHs are already in place at the peak of the starburst is in line with the expectations of current theory, and provides direct observational evidence for a causal link between the growth of SMBHs and the stellar component of massive galaxies.

\section{Concluding remarks}

These first VLBI detections of SMGs suggest that the AGN may contribute significantly to the bolometric luminosity in some and not at all in others. However, it is important to note that by selecting radio-detected SMGs at the epoch of peak quasar activity with compact radio emission, we are biased towards those SMGs with a significant AGN component. Therefore, while these detections are interesting as direct observational evidence for a connection between extreme starbursts and the build-up of massive SMBHs, they say little about the bolometric contribution of AGN in the broader population of SMGs. Future unbiased surveys with high-sensitivity VLBI arrays should allow the typical AGN fraction in SMGs to be established.

\section{References}

[1] Alexander D. M., Bauer F. E., Chapman S. C., Smail I., Blain A. W., Brandt W. N., Ivison R. J., 2005, ApJ, 632, 736

[2] Biggs A. D., Ivison R. J., 2008, MNRAS, 385, 893

[3] Di Matteo T., Springel V., Hernquist L., 2005, Nat., 433, 604

[4] Hopkins P. F., Hernquist L., Cox T. J., Robertson B., Krause E., 2007, ApJ, 669, 45

[5] Ivison R. J. et al., 2002, MNRAS, 337, 1

[6] Kormendy J., Richstone D., 1995, ARA\&A, 33, 581

[7] Swinbank A. M., Smail I., Chapman S. C., Blain A. W., Ivison R. J., Keel W. C., 2004, ApJ, 617, 64

[8] Younger J. D., et al., 2008, ApJ, 688, 59 

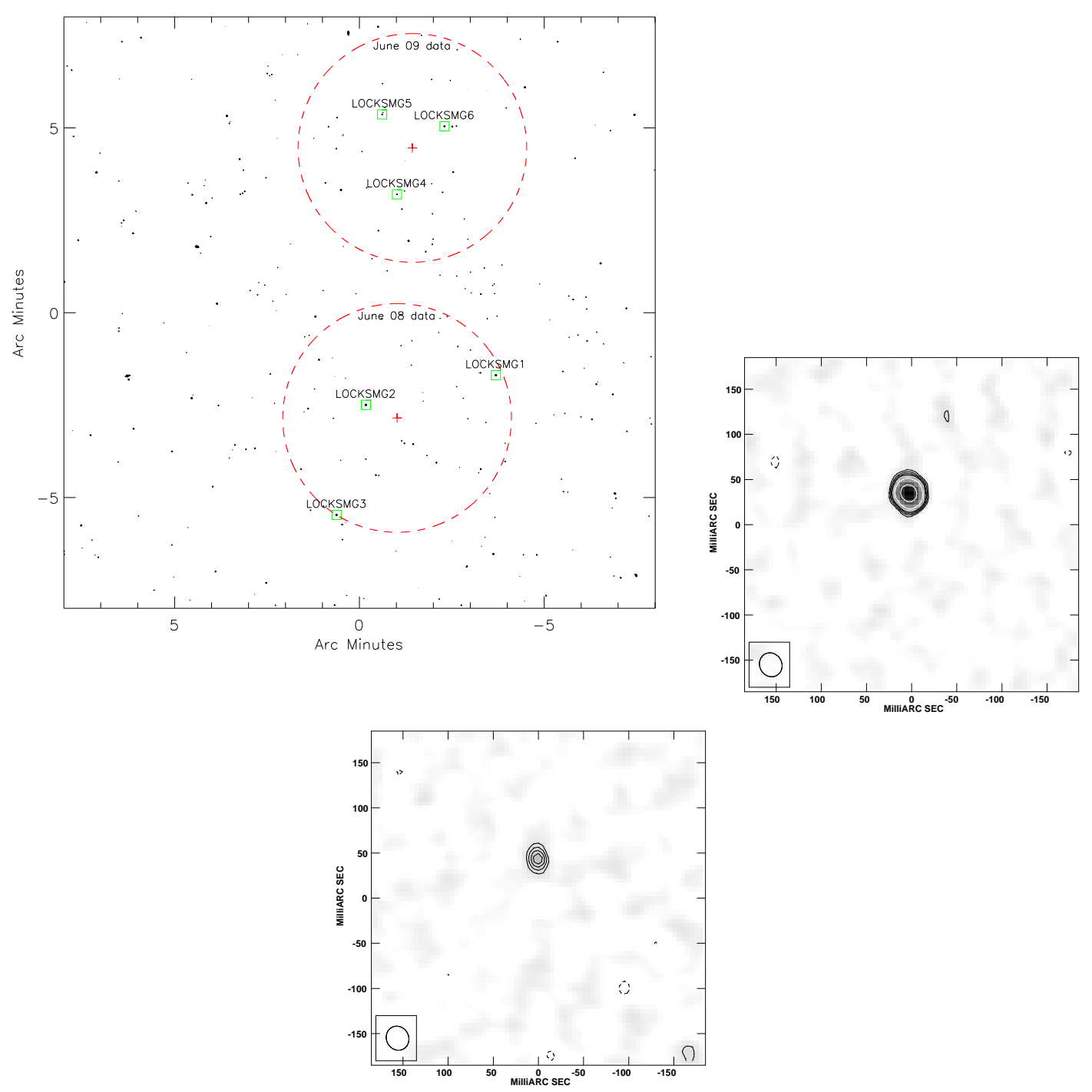

Figure 1: Top-left: VLA 1.4-GHz map of the Lockman Hole. Radio sources that have been identified as submillimetre emitters and which have or are being targeted by the EVN are marked with squares. The dashed circles indicate the 6-arcmin (FWHM) primary beam of the 100-m Effelsberg telescope at the observed and to-be observed epochs. Bottom-left: Naturally-weighted CLEAN maps of LOCKSMG2 (top) and LOCKSMG3 (bottom), the two detections from the June 08 data. The $1-\sigma$ rms noise in the images is $10 \mu \mathrm{Jy} / \mathrm{beam}$, the beam size is $30 \mathrm{mas}^{2}$ and contours are plotted at $-3,3,4,5,6,10,20 \sigma$. 